\title{
Differential organ toxicity in the adult zebra fish following exposure to acute sub-lethal doses of $10 \mathrm{~nm}$ silver nanoparticles
}

\author{
Janice L Speshock ${ }^{*}$, Nathan Elrod ${ }^{1}$, D Keith Sadoski ${ }^{1}$, Elizabeth Maurer ${ }^{2}$, Laura K Braydich-Stolle ${ }^{2}$, Jeff Brady ${ }^{3,4}$ and Saber Hussain $^{2}$ \\ ${ }^{1}$ Department of Biological Sciences, Tarleton State University, Stephenville, TX 76402, USA \\ ${ }^{2}$ Air Force Research Laboratories Human Effectiveness Directorate, Wright-Patterson Air Force Base, OH 45433, USA \\ ${ }^{3}$ Department of Wildlife, Sustainability, and Ecosystem Sciences, Tarleton State University, Stephenville, TX 76402, USA \\ ${ }^{4}$ Texas A\&M Agri Life Research and Extension, Stephenville, TX 76401, USA
}

\begin{abstract}
The novel anti-microbial properties of silver nanomaterials have made them desirable for use in health and medicine. However, prior to in vivo usage of any material, a thorough understanding of bio-distribution and potential toxicity is required. The majority of the toxicological data for silver nanoparticles (Ag-NPs) is still primarily from single-cell culture models, which can often be misleading and lack in vivo correlation. To develop a greater understanding of the fate and interactions of Ag-NPs within a living organism, we administered $\mathrm{Ag}$-NPs to adult zebrafish via the intramuscular route, a common medical administration site, and tracked the fate and toxicity. Although at the doses used in the study there was no death, silver was detected in many of the major organs of the zebrafish, indicating that dispersion occurred from the site of inoculation. The heart, gall bladder, spleen, and kidney all demonstrated varying degrees of damage due to the nanoparticles, and further evaluation identified inflammation caused by the presence of silver as the cause, suggesting that toxicity may be a factor if Ag-NPs are used in a clinical setting.
\end{abstract}

\section{Introduction}

Silver nanoparticles (Ag-NPs) have increased catalytic, sensing, and anti-microbial capabilities, which have made them ideal candidates to be used in many important fields of science including medical diagnosis and detection, environmental wastewater cleanup and food purification and preservation [1]. However, despite their fascinating potential, there remains a great deal of concern about their toxicological properties and potential negative human and/or environmental impact [1]. Therefore, the scientific community needs to develop a greater foundation into the knowledge of the biological interactions of nanomaterials with humans, plants, and animals, and determine any negative ramifications of these interactions prior to wide-scale use and incorporation of AgNPs into consumer products and applications.

Since zebrafish (Danio rerio) share many protein orthologs with humans and contain a comparable immune system, their use has increased as a novel tool in fields such as development, genetics, toxicology, and immunology, due to their small size, expanding biological tool base, fully sequenced genome, and low cost of breeding and maintenance $[2,3]$. When compared to mammalian models, these properties also make them an ideal candidate for a rapid preliminary screening tool for toxicological assessment $[2,3]$ due to the low cost of housing, use, and breeding.

Although some of the biological barriers found in humans do not exist in zebrafish, the use of these small, inexpensive animals allows for a greater understanding of bio-distribution, bio-localization, and excretion, which more traditional methods such as cell lines lack. It is feasible to use much larger samples sizes for trial groups since the cost of zebrafish, especially when bred in the facility, are very low.
Furthermore, although they may lack some of the fundamental tools of toxicological assessment found in rodent models, the zebrafish model is very useful for preliminary assessment of lethality, distribution, ionic dissociation, and intercellular communication, which has been determined to impact toxicological assessments [4]. These parameters need to be evaluated using in vivo models, and the zebrafish provides an inexpensive alternative for early screening.

While most of the nanotoxicity studies have focused on using zebrafish embryos, in this current study, adult zebrafish were used as a system to assess the potential fate of Ag-NPs in a clinical scenario following a standard intramuscular injection. Following Ag-NPs administration at sub-lethal doses, the bio-distribution, deposition in major organs, and subsequent toxicity was evaluated for different concentrations over time. Neither of the concentrations tested ( 1 or 5 milligrams per kilogram $(\mathrm{mg} / \mathrm{kg}$ ) of body weight) were lethal to the zebrafish and caused no observable distress to the fish following an acute $24 \mathrm{~h}$ exposure. It was hypothesized that a non-lethal dose of silver nanoparticles will disseminate from the initial site of injection to the major organs and result in acute toxicity to the tissues. As predicted, tissue damage and inflammation as well as accumulation of the Ag-NPs was evident in the heart, gall bladder, lung, and kidneys of the zebrafish indicating that increased exposure could result in more severe damage to vital organs of the fish.

Correspondence to: Janice L. Speshock, Ph.D, Tarleton State University, 201 St. Felix Street, T0100, Stephenville, TX 76402, USA, Tel: (254) 968-9341, E-mail: speshock@tarleton.edu

Received: March 11, 2016; Accepted: April 13, 2016; Published: April 18, 2016 


\section{Methods}

\section{Zebrafish care and housing}

Zebrafish were housed in a climate-controlled animal facility with 14/10 hour light/dark cycles as approved by Tarleton State University's institutional animal care and usage committee. The zebrafish were between 3-3.5 centimeters in length with an average weight of $0.01 \mathrm{~kg}$, and were obtained from two different suppliers to maintain diversity (Carolina Biological Supply Company, Burlington, NC and Brian's Aquarium Supply, Stephenville, TX). They were housed in groups of 20 in 10-gallon aquariums filled with filtered tap water, supplemented with aquarium salts as per the ZFIN (Zebrafish Information Network) guide [5] with daily chemical testing to ensure desired $\mathrm{pH}$, nitrate, and nitrite levels. The fish were fed three times daily with flake fish food using an automatic feeder, while food was withheld from the fish during the inoculation procedures, which were approximately 8 hours.

\section{Nanomaterials}

Silver nanoparticles (Ag-NPs) selected for this study were the BioPure $10 \mathrm{~nm} \pm 2 \mathrm{~nm}$ coated with a $40 \mathrm{kD}$ polyvinylpyrrolidone (PVP) surface polymer to ensure monodispersity and limit agglomeration (nanoComposix, San Diego, CA). There were two different concentrations used in this study, a concentration of $5 \mathrm{mg} /$ $\mathrm{kg}$, which is often observed to be toxic in cell lines [6] was selected as a high dose and a low dose of $1 \mathrm{mg} / \mathrm{kg}$, which does not cause in vitro cell cytotoxicity [6] was also studied. Both doses were reconstituted in sterile phosphate-buffered saline (PBS). The PVP control contained the purified compound diluted in PBS at the same level as the amount of PVP used to create the high dose of Ag-NP used in this study, which correlates to $0.0005 \%$ PVP.

\section{Zebrafish inoculations}

All treatment protocols were approved by the Institutional Animal Care and Usage Committee prior to initiation. For the treatments, the zebrafish were moved 3 per group into $600 \mathrm{~mL}$ sterile glass beakers containing $250 \mathrm{~mL}$ of fresh water. For the inoculations, an individual zebrafish was anesthetized using $168 \mu \mathrm{g} / \mathrm{mL}$ tris-buffered tricaine (3-aminobenzoic acid ethylester, $\mathrm{pH}$ 7.0; Sigma, St. Louis, MO) diluted in sterile water [2]. Once the fish was sedated, the dorsal muscle left of center was sterilized with an alcohol wipe and $10 \mu$ of sample was injected into the dorsal muscle just behind the dorsal fin using an ultrafine, sterile insulin syringe (BD, East Rutherford, NJ). Intramuscular injections were selected as an exposure method to ensure that the entire sample was internalized in vivo. Once the zebrafish was inoculated, it was returned to the beaker of aquarium water and was monitored for 24 hours. At 24 hours post-exposure, the zebrafish were euthanized via submersion in a beaker of ice cold slush made from sterile water and ice and the heart, spleen, gallbladder, and kidneys were aseptically removed, examined, and processed as needed. For fish whose organs were to be used for real time PCR analysis, upon removal the organs were placed into RNAlater solution (Qiagen Corporation, Germantown, MD) and frozen at $-80^{\circ} \mathrm{C}$. The organs that were going to be analyzed for inductively coupled plasma mass spectrophotometry (ICP-MS) or cytotoxicity were homogenized and were then stored at $4^{\circ} \mathrm{C}$. The organs for Transmission Electron Microscopy (TEM) or histology were immediately placed into a $2 \%$ paraformaldehyde/2.5\% gluteraldehyde solution for fixation (Electron Microscopy Sciences, Hatfield, PA).

\section{Inductively-Coupled Plasma Mass Spectrometery}

The presence or absence of silver in the organs was assessed using
Inductively-Coupled Plasma Mass Spectrometry (ICP-MS). The organ samples were acid digested in a 3:1 ratio of hydrochloric acid to nitric acid with $10 \mathrm{~mL}$ of internal standard mix (Perkin Elmer, MA, USA) for an end concentration of $3 \% \mathrm{HCl}$ and $1 \% \mathrm{HNO}_{3}$ in a volume of 5 $\mathrm{mL}$. The ICP-MS procedure was conducted in standard mode with 20 sweeps per reading, at 1 reading per replicate, and 3 replicates per sample with a dwell time of $100 \mathrm{msec}$ using a Perkin Elmer ICP-MS 300D. A calibration curve was obtained using four silver standard solutions, and the addition of an internal standard was done to ensure that no interferences were occurring [7].

\section{Transmission electron microscopy}

For transmission electron microscopy (TEM) analysis, the individual organs were excised and fixed in a $\%$ paraformaldehyde $/ 2.5 \%$ glutaraldehyde mixture then washed and stained with $1 \%$ osmium tetroxide (Electron Microscopy Sciences, Hatfield, PA). Following the staining the organs were dehydrated with the addition of successively higher concentrations of EtOH. After the samples had been dehydrated they were embedded in LR white medium grade resin and cured overnight in a vacuum oven at $60^{\circ} \mathrm{C},-15 \mathrm{inHg}$. The embedded organs were sectioned on an ultramicrotome (Leica Ultracut) with a thickness of $\sim 50 \mathrm{~nm}$ and collected onto carbon-formvar-coated copper TEM grids. Brightfield TEM micrographs were obtained using the Hitachi H-7600 TEM to determine the presence or absence of intact nanomaterials in the organs [7].

\section{Cytotoxicity}

Although they are designed for cell culture, an attempt at adapting the CytoTox96 (Lactate Dehydrogenase; LDH) and CellTiter 96 AQueous One (MTS) kits from Promega (Madison, WI) for whole organ use was performed. Briefly, the organs were homogenized in sterile PBS and the organ homogenates were used in each of the LDH and MTS kits as per the manufacturer's instructions instead of typical cell culture media. The LDH kit was successful, but the MTS kit was not.

\section{Gene expression analysis}

Organ homogenates stored in RNAlater were homogenized, passed through a 26 gauge syringe for tissue disruption, and then solid phase extracted on a Qiagen RNeasy column (Qiagen Corporation, Germantown, MD) as per the manufacturer's instructions, with optional DNase digestion step, to purify RNA. The RNA was quantified using a Qubit (Life Technologies, Carlsbad, CA) with a high sensitivity RNA fluorometric assay kit. Fifty nanograms of RNA were reverse transcribed to cDNA using the Biorad iScript cDNA synthesis kit (Biorad, Hercules, CA). The cDNA was used to perform quantitative real time PCR (qRT-PCR) using the Roche Light Cycler 480 system (Basel, Switzerland) and custom-designed primers synthesized by Integrated DNA Technologies (Coralville, IA). Amplification reactions $(5 \mathrm{~L})$ contained $1 \mathrm{~L} \mathrm{cDNA}, 1 \mathrm{~L}$ sterile molecular grade water, $0.25 \mathrm{~L}$ forward and reverse primer mix (10 M of each), $0.25 \mathrm{~L} 10 \mathrm{M}$ probe stock, and 2.5 L 2X probe master mix as per Painter et al. [8]. The fold change was determined using the 2(-Delta Delta $\mathrm{C}(\mathrm{T})$ ) method over control primer beta-actin [9] Primer sequences are supplied in Table 1 of the supplemental section.

\section{Statistical analysis}

The Student's t test was used to confirm the significant up- or downregulation of gene expression with a $\mathrm{p}$ value less than 0.05 considered to be significant. 
Table 1. Primer sequences.

\begin{tabular}{|c|l|l|}
\hline Target & Forward Primer & Reverse Primer \\
\hline *Interleukin-1 beta & 5'-aacctgtacctggcctgttg-3' & 5'-atctccaccatctgcgaatc-3' \\
\hline Tumor Necrosis Factor alpha & 5'-gcgctttctgaatcctacg-3' & 5'-tgcccagtctgtctccttct-3' \\
\hline Interleukin-6 & 5'-gtccccgtgttcagcagtat-3' & 5'-ctgatcctgaccccttcaaa-3' \\
\hline Caspase-3 & 5'-gccactgcatcatcatcaac-3' & 5'-taatctgcgcaactgtctgg-3' \\
\hline Caspase-6 & 5'-actgccttgtctgcgtcttt-3' & 5'-gtccatcggagtcacaggat-3' \\
\hline Caspase-8 & 5'-ggaggaattgtgtcccaaga-3' & 5'-aaacggaagattgtccaacg-3' \\
\hline Caspase-9 & 5'-agggcaagcccaagttattt-3' & 5'-cagacgctgtgctgagagag-3' \\
\hline FADD & 5'-ctttgtctgctgcctcatca-3' & 5'-ggcattggaacacttcctgt-3' \\
\hline *Interleukin-10 & 5'-atttgtggagggctttcctt-3' & 5'-agagctgttggcagaatggt-3' \\
\hline *Beta Actin & 5'-gtgcccatctacgagggtta-3' & 5'-tctcagctgtggtggtgaag-3' \\
\hline & & \\
\hline
\end{tabular}

*Roca et al. [27]

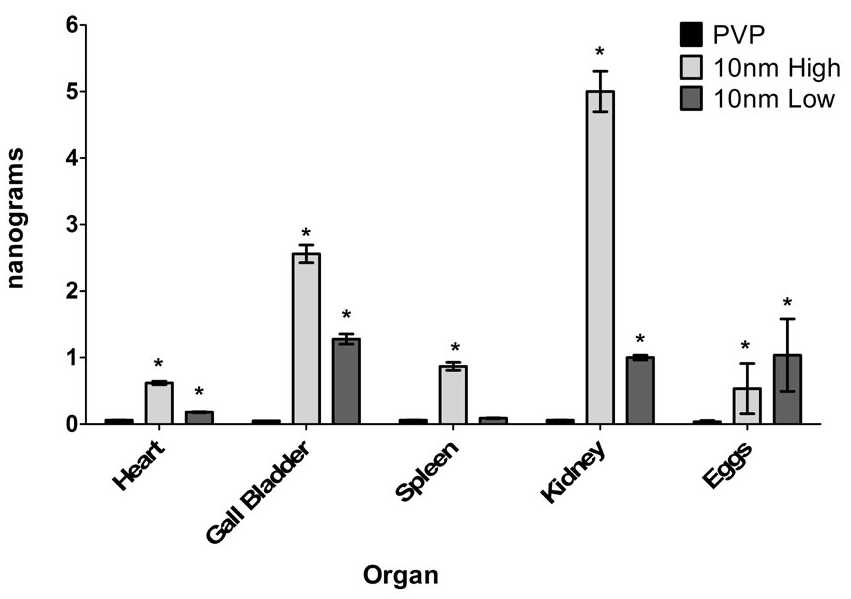

Figure 1. Silver concentration in the organs. Organs or eggs $(n=3)$ were assayed for their silver content using ICP-MS following high ( $5 \mathrm{mg} / \mathrm{kg}$ ) or low $(1 \mathrm{mg} / \mathrm{kg})$ Ag-NP exposure. * denotes significant increase over the PVP control $(\mathrm{p}<0.05)$.

\section{Results}

\section{Silver concentration in organs}

Macroscopic observation of the organs demonstrating altered anatomical appearance suggested that the Ag-NPs were able to disseminate from their point of origin, the dorsal muscle, and interact with various organs throughout the fish. Individual organs excised from fish exposed to $5 \mathrm{mg} / \mathrm{kg} 10 \mathrm{~nm} \mathrm{Ag-NP} \mathrm{(10nm} \mathrm{High),} 1 \mathrm{mg} / \mathrm{kg}$ $10 \mathrm{~nm}$ Ag-NP (10 nm Low), or the capping agent control (PVP) were analyzed using inductively-coupled plasma mass spectrometry (ICPMS) to determine the concentration of silver in each organ (Figure 1). Four organs (heart, gallbladder, spleen, and kidney) were digested and analyzed for silver content and a significant amount of silver was detected in all organs when the fish were treated with the $5 \mathrm{mg} / \mathrm{kg}$ dose and the heart, gallbladder, and kidney for the $1 \mathrm{mg} / \mathrm{kg}$ dose (Figure 1). This confirms that the Ag-NPs do disseminate throughout the body and deposit in major organs. As expected, the higher inoculum of AgNP resulted in higher silver content in each organ. Three pregnant females for each treatment were included to determine if the offspring may be affected by parental exposure to Ag-NPs and both the high and low dose of Ag-NPs caused detectable levels of silver in the eggs of the pregnant females (Figure 1). ICP-MS was also used to detect the presence of silver in the water housing the treated zebrafish and only the $5 \mathrm{mg} / \mathrm{kg}$ dose had trace amounts of silver present in the water $(0.230 \mathrm{ng} / \mathrm{ml})$ after 24 hours of exposure suggesting that the silver was associated with the fish and not being excreted out.

\section{Silver nanoparticles in the organs}

Although silver was detected in each organ via ICP-MS and differential organ toxicity was observed following silver exposure, there was little observation of intact Ag-NPs (Figure 2). The only organ where Ag-NPs were detected was the heart (Figure 2A-C) where a small cluster of particles was found ranging in size from 3-6 nm (Figure 2C). Although organ pathological changes were observed in the gallbladder (Figure 2D), the spleen (Figure 2E), and the kidney (Figure 2F) in the zebrafish exposed to the high dose of Ag-NPs, a thorough examination of the organs did not result in the location of any Ag-NPs. The PBS control organ TEM images are included in Figure $\mathrm{S} 1$ for reference.

\section{Lactate Dehydrogenase assay for membrane integrity}

LDH is a cytoplasmic enzyme that is only extracellular when the cellular membrane is compromised. The heart demonstrated no change in $\mathrm{LDH}$ levels between Ag-NP treatment and control, indicating that the membrane integrity remained and no necrosis occurred (Figure 3A). In contrast, a large increase in extracellular LDH levels was detected in the splenocytes (Figure 3C) demonstrating that the Ag-NPs are causing tissue pathology to the spleen. The LDH levels in the gallbladder (Figure 3B) and kidney (Figure 3D) were lower than control suggesting that the intracellular LDH has been released, just at an earlier time point than assayed since the estimated half-life of extracellular LDH according to the manufacturer is 9 hours. The PVP capping agent appeared to display a similar degree of cytotoxicity as the PVP-coated Ag-NPs in the kidney only, while no other assayed organ was affected (Figure 3). An MTS assay was also attempted to evaluate cell viability, but it did not work effectively with whole organs (data not shown).

\section{Inflammation in the organs following Ag-NP exposure}

The pro-inflammatory cytokines interleukins 1 and 6 (IL-1, IL-6) and tumor necrosis factor alpha (TNFa) were assessed for changes following Ag-NP exposure using qRT-PCR (Figure 4). IL-1 was significantly up-regulated in all organs for the high dose of exposure to Ag-NPs (Figure 4). The low dose and PVP demonstrated a trend
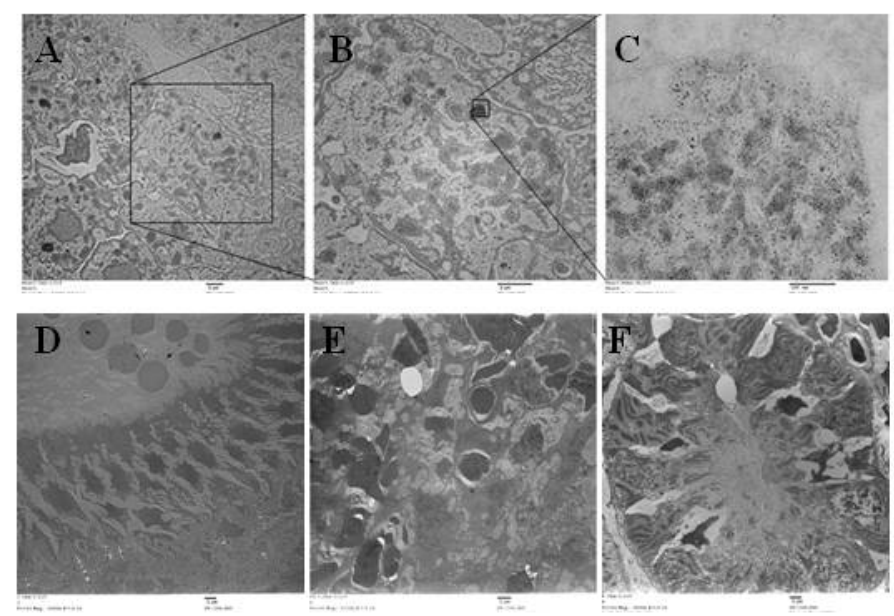

Figure 2. Transmission electron micrographs of organs. Figures $2 \mathrm{~A}-\mathrm{C}$ depicts the presence of Ag-NPs in the heart with the square section being enhanced in $2 \mathrm{~B}$ and then $2 \mathrm{C}$. Figures 2D-F illustrate representative samples of the low dose Ag-NP treated gallbladder, spleen, and kidney, respectfully. There were no Ag-NPs found in any of these organs at the low or high dose of Ag-NPs. The high dose TEM images were not as clear due to the poor quality of the excised organs. The scale bars on all images except for $2 \mathrm{C}$ are set at 1 micron. Figure $2 \mathrm{C}$ scale is at $500 \mathrm{~nm}$. 

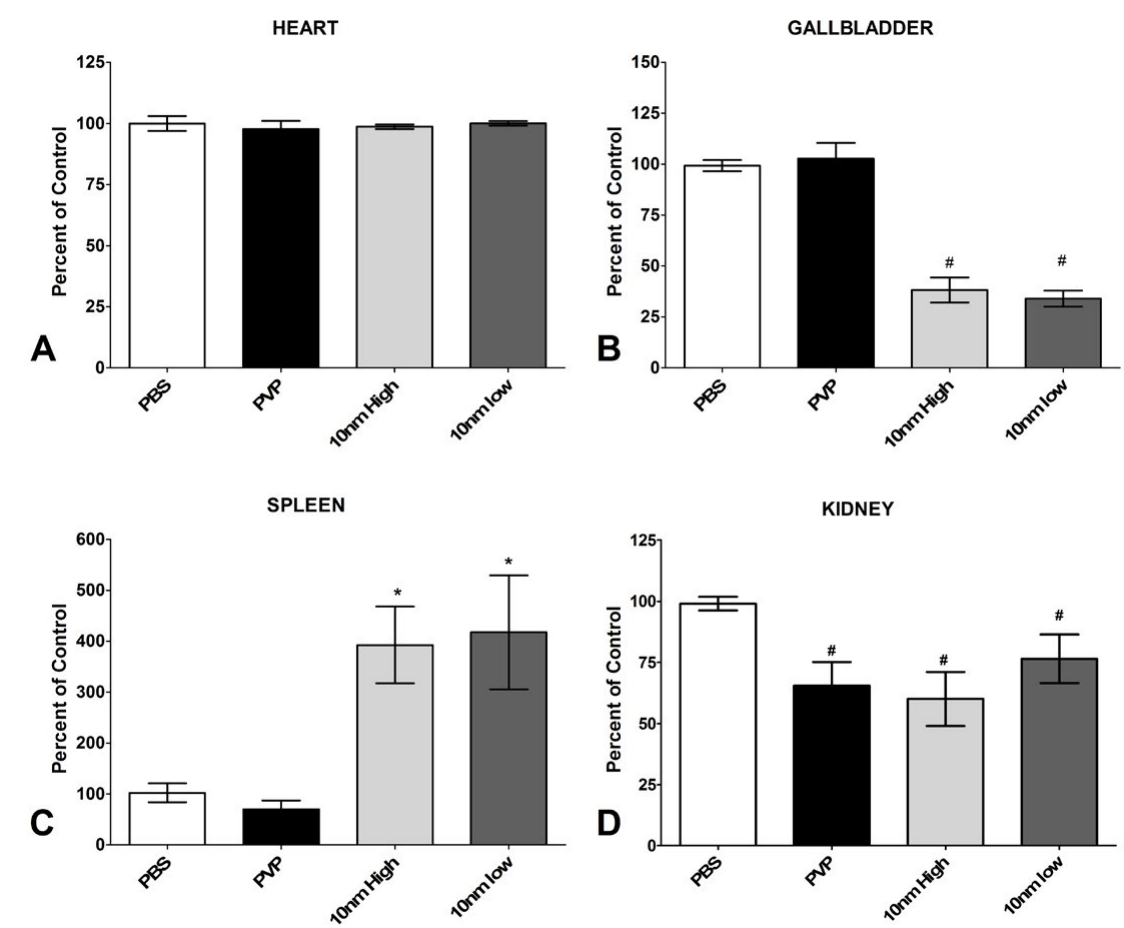

Figure 3. Extracellular LDH concentrations in the organs. The extracellular LDH leakage was assayed following high (5 mg/kg) or low (1 mg/kg) Ag-NP exposure using a commerciallyavailable kit and were reported as percent of control, which was normalized to $100 \%$. ${ }^{*}$ denotes significant increase over the saline (PBS) control and \# denotes significant decrease under control $(\mathrm{p}<0.05)$.

towards increase in the heart and gallbladder (Figure 4A and B), but the results were not significant. For the spleen and kidney, the low dose of Ag-NP exposure stimulated significant IL-1 production, but PVP did not (Figure 4C and D). TNFa demonstrated an up-regulated trend, but was only significantly up-regulated in the spleen and kidney for zebrafish receiving the low dose exposure (Figure 4C and D). IL-6 did not form a discernable pattern of activation and therefore does not appear to greatly contribute to Ag-NP-induced pathology (Figure 4).

\section{Caspase activity}

The capping agent PVP showed some signs of toxicity towards the heart tissue due to the up-regulation of caspases 6 and 9, which indicate activation of the intrinsic pathway of apoptosis (Figure 5A). These two caspases in addition to caspase 8 were up-regulated in the high dose treatment of nanoparticles in the heart, but only caspase 6 was up-regulated in the low dose treatment (Figure 5A). However, due to the lack of cytotoxicity data in Figure 3, it is hypothesized that the pro-caspase was being synthesized and perhaps not cleaved and activated. An up-regulation of caspase 6 was observed in both treatment groups of the gallbladder, but not in the capping agent or control (Figure 5B). No other caspases were up-regulated at this time point and due to the late activation of caspase 6 in the caspase cascade, this confirms the LDH data obtained for the gallbladder (Figure 3B) suggesting that the damage to the organ occurred at an earlier time point. The high dose of Ag-NPs up-regulated the activation of caspase 8 in the spleen, which is necessary for the extrinsic pathway of apoptosis, and a corresponding increase in Fas-associated death domain (FADD; Figure S2) and TNF-alpha (Figure 4C). Interestingly, the low dose exposure to the spleen had a reduction in the levels of caspase 8 (Figure 5C), but had even more dramatic increases in FADD (Figure S2) and TNF-alpha (Figure 4C). The downstream caspases 6 and 8 are however up-regulated suggesting that perhaps the activation is further along in the low dose treatment than the high (Figure 4C). The significant up-regulation of caspase 9 indicates that it is the intrinsic pathway of apoptosis that causes pathological changes to the kidney, and the upregulation in the PVP control implicates the capping agent as a strong contributor, although the presence of the silver did further increase the up-regulation (Figure 5D).

\section{Discussion}

Although previous studies have indicated that nanosilver induces mortality at very low doses in aquatic animals $[10,11]$, the formulation of nanosilver that was used in this study was not lethal to the zebrafish via intramuscular inoculation or administration to the water at 1 or 5 $\mathrm{mg} / \mathrm{kg}$ for the one week observation period. Thus this study confirms the challenge with nanomaterial toxicological assessments that every particle generated must be assessed individually and generalizations cannot be made (a detailed review of these challenges is presented by Hussain et al. Bilberg et al. [10, 12] also capped their Ag-NPs with PVP, but observed a lethal dose at $50 \%$ at the concentration of $84 \mu \mathrm{g} / \mathrm{L}$, nearly 1000 -fold lower than our dose, when administered to the aquarium water [10]. The size of the particle used in the Bilberg study was much larger $(81 \mathrm{~nm})$ than used here $(10 \mathrm{~nm})$, but a majority of cell culture experiments using nanosilver suggest that a larger particle should be less toxic than the smaller nanoparticles $[10,13]$. Therefore the synthesis method, which cannot be commented on since commercial particles were utilized here, or perhaps the water chemistry may be impacting the toxicity of the nanoparticles to the zebra fish.

The $10 \mathrm{~nm}$ PVP-coated Ag-NPs obtained for this study were capable of dissemination from the point of inoculation and did appear to affect many of the major organs. Histological examination of the 

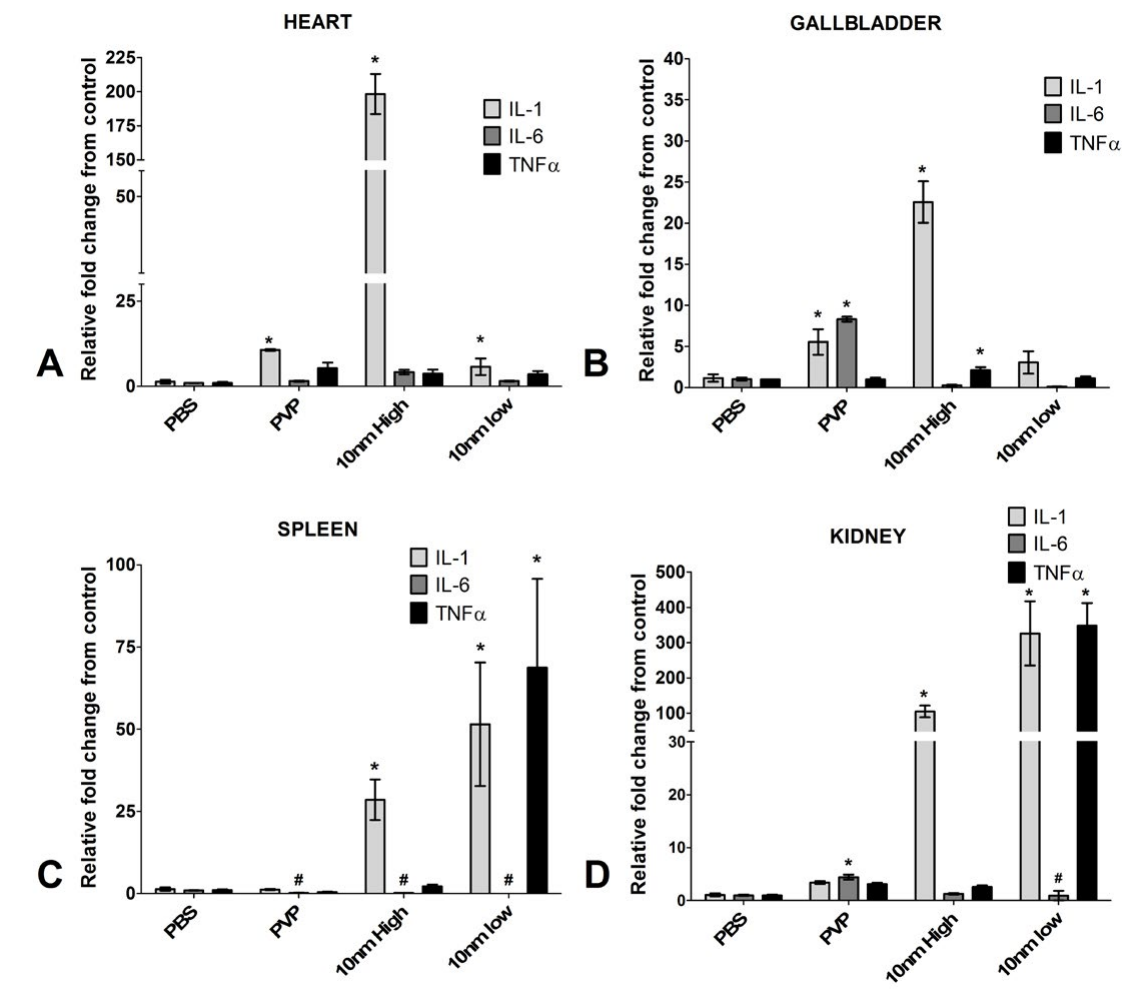

Figure 4. Gene expression of pro-inflammatory cytokines. Quantitative reverse transcriptase PCR was used to determine activation or inhibition of the pro-inflammatory cytokines following high $(5 \mathrm{mg} / \mathrm{kg}$ ) or low $(1 \mathrm{mg} / \mathrm{kg})$ Ag-NP exposure and were reported as relative fold change from control (PBS), which was normalized to 1. * denotes significant increase over the saline (PBS) control and \# denotes significant decrease under control $(\mathrm{p}<0.05)$.

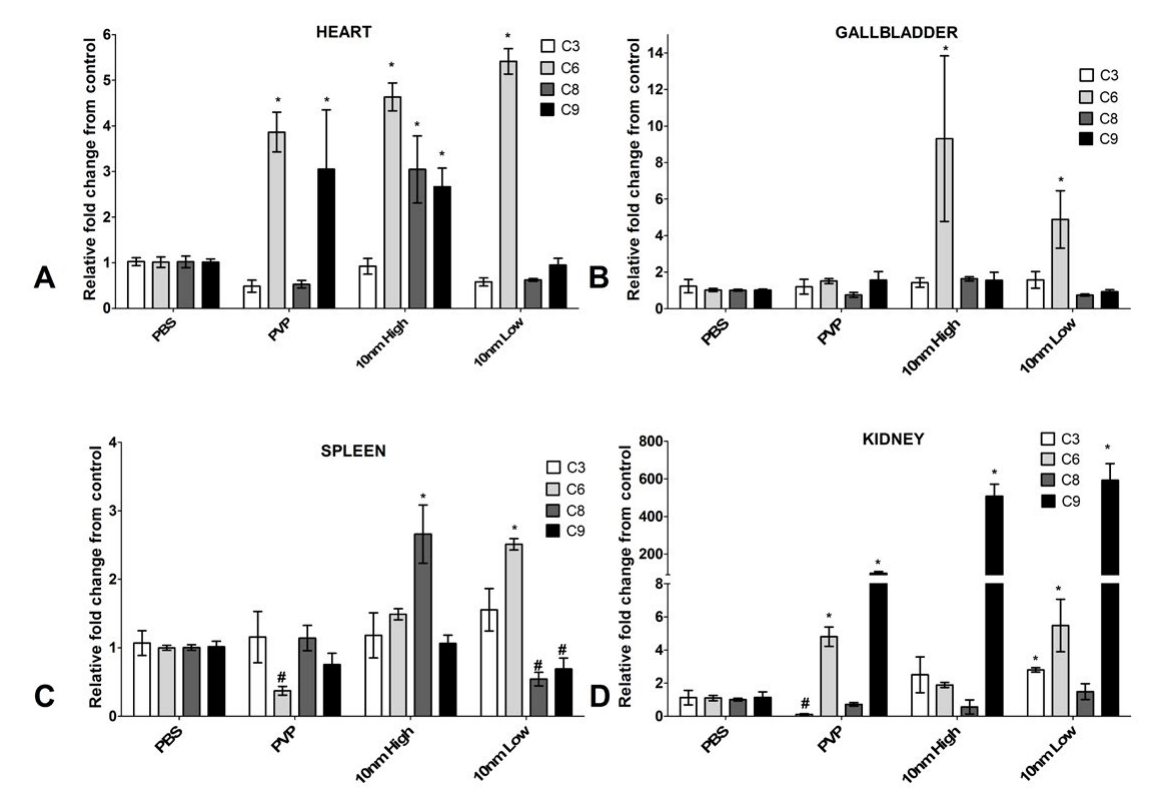

Figure 5. Gene expression of cellular caspases. Quantitative reverse transcriptase PCR was used to determine activation or inhibition of apoptotic caspases following high (5 mg/kg) or low $\left(1 \mathrm{mg} / \mathrm{kg}\right.$ ) Ag-NP exposure and were reported as relative fold change from control (PBS), which was normalized to $1 .{ }^{*}$ denotes significant increase over the saline (PBS) control and \# denotes significant decrease under control $(\mathrm{p}<0.05)$.

brains of the $5 \mathrm{mg} / \mathrm{kg}$ dose suggests mild hemorrhaging and swelling may have occurred (Figure S3C) although the pathology did not appear to have a dramatic effect on the fish since mortality was not observed. Molecular analyses and microscopy were not performed on brains due to the difficulty of removing them from exposed fish. The other organs that were less challenging to remove were assessed for the presence of silver and specifically Ag-NPs, changes in inflammatory state, and any indications of cellular apoptosis or tissue necrosis. 
The heart displayed the most unique response to an intramuscular inoculation with Ag-NPs. Although it had one of the lowest levels of silver following exposure with either dose, the heart was the only organ where discernable nanoparticles were found. The nanoparticles appeared to aggregate to particular areas of the heart and were observed to be slightly smaller in size $(3-6 \mathrm{~nm})$. Asharani et al. too observed a deposition of Ag-NPs in the zebra fish heart when examining embryos exposed to $5-20 \mathrm{~nm}$ particles, suggesting that the heart may be a target organ for these materials [14]. Interestingly, although the heart was the only organ where intact nanoparticles were found, it was also the organ with the least toxicity. Dissociation of silver into its ionic form has been suggested to contribute more to toxic effects than the intact nanoparticles, which could describe the results observed in these zebrafish [14]. A recent study using silver nanospheres demonstrated that a decrease in cell viability occurred as the ionic concentration increased [15]. Since the kidney, gallbladder, and spleen all had detectable silver, but no visible particles, and they had the most observable pathology, we hypothesize that the Ag-NPs may have dissociated into silver ions in these organs. The intracellular LDH levels were comparable to control, and although the caspase levels were significantly elevated, they were far lower than other organs, and appeared to be due to the PVP capping agent rather than the nanoparticles themselves. There was a significant up-regulation of the pro-inflammatory cytokine interleukin-1 (IL-1), but the heart was the only organ where a corresponding increase of the anti-inflammatory cytokine IL-10 was also observed (Figure S4), suggesting a regulation of inflammation [16].

Molecular analyses were performed on the gallbladder due to the dramatic observable change in color between the Ag-NP-exposed and PVP or mock-exposed zebrafish. The loss of characteristic green color suggests a loss of bile either through underproduction by the liver or leakage from the gallbladder. The LDH data indicates that the membrane integrity of the cells comprising the gallbladder is highly compromised and therefore bile leakage is a more likely scenario. The gallbladder had elevated silver levels, but no intact Ag-NPs were found. The high concentration of enzymes in the organ could be contributing to the breakdown of the nanoparticles into an ionic silver form. The upregulation of IL-1, without a correlating increase of anti-inflammatory cytokines, indicates a great deal of inflammation in the organ postsilver exposure and some of the inflammation appears to be due to the PVP capping agent itself. The caspase- 6 was significantly up-regulated in the gallbladders suggesting initiation of apoptosis, but since the downstream caspases- 3 , and -8 were never activated, it is most likely that any caspase- 6 protein produced was degraded [17].

The spleen had the least notable histopathological changes and lowest silver levels of the major organs assayed suggesting that either the nanoparticles are not entering the bloodstream or they are filtered out of the blood prior to entry into the spleen. Even though the damage was not readily apparent, due to the substantial increase in extracellular $\mathrm{LDH}$, pro-inflammatory cytokines, caspases, and the Fas-associated protein with death domain, FADD (Figure S2), splenocytes are likely being damaged by the presence of the silver through the extrinsic apoptotic pathway $[18,19]$. The terminal caspase, caspase- 3 , which initiates apoptosis was not up-regulated in the spleen, or any other organ with apparent cytotoxicity, suggesting that either the zebrafish utilize a different caspase cascade or the primers developed were not sufficient for adequate detection [20]. The up-regulation of caspase-8 could alternatively be stimulating the processing of pro-interluekin1beta to the biologically active form in a unique NLRP3 inflammasome- independent manner only observed with the activation of dectin-1 by fungi and mycobacteria [21].

Being that it is the major filtration organ of the body, it is not very surprising that the kidney had the most silver deposition and most cytotoxicity. Perhaps the most concerning finding was the lack of $\mathrm{Ag}$ found in the zebrafish water indicating that once these Ag-NPs reached the kidney, they were retained and generated considerable inflammation to the kidney. Similar to the spleen, a high up-regulation of pro-inflammatory cytokines was observed with no corresponding increase in anti-inflammatory cytokines indicating that the inflammatory response was a contributing factor to the cytotoxicity [22]. However, unlike the spleen, the caspases activated in the kidney suggest an internal activation of the intrinsic pathway of apoptosis with PVP appearing to contribute to the cytotoxicity observed [23]. Since these results were obtained following a single acute exposure, repeated exposure in a chronic setting could potentially lead to kidney failure.

One of the more surprising, yet more alarming, findings of this study was that the Ag-NPs were able to enter into the eggs although the TEM micrographs were inconclusive (image not shown) so it cannot be determined if the nanoparticles remained intact. However, this suggests that adult exposure to Ag-NPs could lead to defects in progeny since many studies have examined the effects of Ag-NPs on zebrafish embryos and much of the data is negative. Asharani et al. demonstrated that 5-20 nm silver caused increased mortality and decreased hatch rate of zebrafish embryos and accumulation of the Ag-NPs in the heart and brain of the developing zebrafish [14] In a more recent study, it was demonstrated that even exposure to larger Ag-NPs (approximately $97 \mathrm{~nm}$ ) results in toxicological effects and phenotypic abnormalities in zebrafish embryos at various stages of development [24]. Powers et al. provided a comparison assessment of the coatings of Ag-NPs as well as dissociated ions, determining that there are many factors that can determine how the silver affects the embryos [25]. Another study that used commercially-available $10 \mathrm{~nm}$ Ag-NPs had similar results in the embryo that we observed with the adult zebrafish in that they did not observe any increase in mortality of the zebrafish embryos but observed substantial changes in gene regulation using HighThroughput SuperSAGE with next-generation sequencing especially with silver ions [26] confirming our hypotheses that lack of mortality does not necessarily correlate to lack of toxicity and that all particles must be assessed individually [27].

\section{Conclusion}

The use of inexpensive biological tools for preliminary screening of nanomaterials is advantageous due to the many different synthesis techniques, core materials, and capping agents being utilized in research. Zebrafish are an ideal model for initial assessments of toxicity for chemicals and other materials because they are inexpensive and there is a rapidly growing database of tools available, which allow for adequate assessments for whole-organism exposure scenarios. Our results demonstrated the simple observation of live versus dead cannot be the only parameter used when deciding whether or not to use these materials in medical applications since repeated exposure will result in cumulative damage that can ultimately cause severe organ trauma. In addition, the up-regulation of inflammation observed throughout the organism could impact the ability of the immune system to combat disease or could result in heightened pathology due to infectious agents. Silver nanomaterials do have many attractive properties and therefore their medicinal use should be assessed further, but the availability of information on the potential side-effects should also be 
well understood prior to their wide-spread use.

\section{Acknowledgements}

We would like to thank the Tarleton State University Office of Student Research and Creative Activities for funds to complete this project and for stipends for Mr. Elrod and Mr. Sadoski. We would also like to acknowledge the assistance of AML laboratories (Baltimore, MD) for the histological processing of the zebra fish.

\section{Declaration of Interest}

The authors have nothing to declare.

\section{References}

1. Majdalawieh A, Kanan MC, El-Kadri O, Kanan SM (2014) Recent advances in gold and silver nanoparticles: synthesis and applications. J Nanosci Nanotechnol 14: 4757 4780.[Crossref]

2. Neely MN, Pfeifer JD, Caparon M (2002) Streptococcus-zebrafish model of bacterial pathogenesis. Infect Immun 70: 3904-3914.[Crossref]

3. Spitsbergen JM, Kent ML (2003) The state of the art of the zebrafish model for toxicology and toxicologic pathology research: Advantages and current limitation. Toxicologic Pathology 31 (Supplement), 62-87

4. Braydich-Stolle LK, Speshock JL, Castle A, Smith M, Hussain SM (2012) Nano-sized aluminum suppressed immune function. ACS Nano 4: 3661.

5. Westerfield M (2000) Thezebrafish book: A guide for the laboratory use of zebrafishDaniorerio. Eugene, OR: University of Oregon Press.

6. de Lima R, Seabra AB, Duran N. 2012. Silver nanoparticles: a brief review of cytotoxicity and genotoxicity of chemically and biogenically synthesized nanoparticles. Journal of Applied Toxicology 32: 867-879.

7. Maurer E, Sharma M, Schlager J, Hussain S (2013) Systematic analysis of silver nanoparticle ionic dissolution by tangential flow filtration: toxicological implications. Nanotoxicology 8: 718-27

8. Painter SM, Pfau RS, Brady JA, McFarland AMS (2013) Quantitative assessment of Naegleriafowleri and Escherichia col concentrations within a Texas Reservoir. Journal of Water and Health 11.2: 346-357.

9. Livak KJ, Schmittgen TD (2001) Analysis of relative gene expression data using realtime quantitative PCR and the 2(-Delta DeltaC(T)) Method. Methods 25: 402-408. [Crossref]

10. Bilberg K, Hovgaard MB, Besenbacher F, Baatrup E (2012) In Vivo Toxicity of Silver Nanoparticles and Silver Ions in Zebrafish (Daniorerio). J Toxicol 2012: 293784. [Crossref]

11. Griffitt RJ, Luo J, Gao J, Bonzongo JC, Barber DS. 2008. Effects of particle composition and species on toxicity of metallic nanomaterials in aquatic organisms. Environmental Toxicology and Chemistry 27: 1972-1978.
12. Hussain SM, Braydich-Stolle LK, Schrand AM, Murdock RC, Yu KO, Mattie DM Schlager JJ, Terrones M. 2009. Toxicity Evaluation for Safe Use of Nanomaterials: Recent Achievements and Technical Challenges. Advanced Materials 21: 1-11.

13. Carlson C, Hussain S, Schrand A, Braydich-Stolle LK, Hess KL, Jones RL, Schlager JJ (2008) Unique cellular interaction of silver nanoparticles: size-dependent generation of reactive oxygen species. Journal of Physical Chemistry B 112: 13608-13619.

14. Asharani PV, Lian Wu Y, Gong Z, Valiyaveettil S (2008) Toxicity of silver nanoparticles in zebrafish models. Nanotechnology 19: 255102. [Crossref]

15. Beer C, Foldbjerg R, Hayashi Y, Sutherland DS, Autrup H (2012) Toxicity of silver nanoparticles - nanoparticle or silver ion? Toxicol Lett 208: 286-292. [Crossref]

16. Sultani M, Stringer A, Bowen J, Gibson R (2012) Anti-inflammatory cytokines: Important immunoregulatory factors contributing to chemotherapy-induced gastrointestinal mucositis. Chemotherapy Research and Practice Article ID 490804

17. Cowling V, Downward J (2002) Caspase-6 is the direct activator of caspase- 8 in the cytochrome c-induced apoptosis pathway: absolute requirement for removal of caspase-6 prodomain. Cell Death Differ 9: 1046-1056. [Crossref]

18. Wang L, Du F, Wang X (2008) TNF-alpha induces two distinct caspase-8 activation pathways. Cell 133: 693-703.

19. Zhao Y, Sui X, Ren H (2010) From procaspase-8 to caspase-8: revisiting structural functions of caspase-8. J Cell Physiol 225: 316-320. [Crossref]

20. Porter AG, Jänicke RU (1999) Emerging roles of caspase-3 in apoptosis. Cell Death Differ 6: 99-104. [Crossref]

21. Gringhuis S, Kaptein TM, Wevers BA, Bart T, van der Vlist M, Boekhout T, Geijtenbeek $\mathrm{T}$ (2012) Dectin-1 is an extracellular pathogen sensor for the induction and processing of IL-1ß via a noncanonical caspase-8 inflammasome. Nature Immunology 13: 246 254

22. Dinarello CA (1991) Interleukin-1 and interleukin-1 antagonism. Blood 77: 1627-1652. [Crossref]

23. Bratton SB, Salvesen GS (2010) Regulation of the Apaf-1-caspase-9 apoptosome. $J$ Cell Sci 123: 3209-3214.[Crossref]

24. Browning LM, Lee KJ, Nallathamby PD, XU XN (2013) Silver nanoparticles incite size and dose-dependent developmental phenotypes and nanotoxicity in zebrafish embryos. Chemical Research in Toxicology 26: 1503-1513.

25. Powers CM, Slotkin TA, Seidler FJ, Badireddy AR, Padilla S (2011) Silver nanoparticles alter zebrafish development and larval behavior: distinct roles for particle size, coating and composition. Neurotoxicol Teratol 33: 708-14.

26. Van Aerle R, Lange A, Moorhouse A, Paszkiewicz K, Ball K, et al. (2013) Molecular mechanisms of toxicity of silver nanoparticles in zebrafish embryos. Environ Sci Technol 47: 8005-8014.[Crossref]

27. Roca FJ, Mulero I, Lopez-Munoz A, Sepulcre MP, Renshaw SA, Meseguer J, Mulero V (2008) Evolution of the inflammatory response in vertebrates: Fish TNF-a is a powerful activator of endothelial cells but hardly activates phagocytes. Journal of Immunology 181: 5071-5081. 